\title{
Anisotropy Constant Required for Thermally Assisted Magnetic Recording
}

\author{
T. Kobayashi, Y. Isowaki, and Y. Fujiwara \\ Graduate School of Engineering, Mie Univ., 1577 Kurimamachiya-cho, Tsu 514-8507, Japan
}

An anisotropy constant $K_{\mathrm{u}}$ required for thermally assisted magnetic recording (TAMR) was calculated. First, a heat transfer simulation was carried out to obtain the thermal gradient of media at the time of writing for the $K_{\mathrm{u}}$ calculation. Both the Curie temperature $T_{\mathrm{c}}$ and $K_{\mathrm{u}}$ should be designated for TAMR media design. $K_{\mathrm{u}}$ is a function of $T_{\text {c. }}$ Therefore, we introduce a parameter $K_{\mathrm{u}} / K_{\mathrm{bulk}}$ that shows the intrinsic ratio of film $K_{\mathrm{u}}$ to bulk $K_{\mathrm{u}}$. From the information stability conditions, we estimated whether or not the media have the potential for TAMR. The limiting factor is the thermal gradient related to the information stability on the trailing side located 1 bit from the writing position during writing and at the adjacent track during rewriting, and the limiting factor determines the minimum $K_{\mathrm{u}} / K_{\text {bulk. }}$. Therefore, the minimum $K_{\mathrm{u}} / K_{\mathrm{bulk}}$ strongly depends on the thermal gradient. Consideration was also given to the dependence of the grain number per bit on $K_{\mathrm{u}} / K_{\mathrm{bulk}}$, the dependence of the standard deviation of the grain size on $K_{\mathrm{u}} / K_{\text {bulk}}$, the dependence of the thickness of the recording layer on $K_{\mathrm{u}} / K_{\text {bulk, }}$, and the dependence of the writing temperature on $K_{\mathrm{u}} / K_{\text {bulk. }}$

Key words: thermally assisted magnetic recording, anisotropy constant, heat transfer simulation, thermal stability factor

\section{Introduction}

Simultaneously satisfying the three conditions of minute magnetic grains, an appropriate thermal stability factor, and suitable coercivity is difficult with high-density magnetic recording ${ }^{1)}$ beyond several Tbits/inch ${ }^{2}$ (Tbpsi). Thermally assisted magnetic recording (TAMR) has been proposed as a way of solving this problem. TAMR is a recording method in which the medium is heated to reduce the coercivity at the time of writing.

We have already proposed a new media design method for 2 Tbpsi TAMR $^{2}$ that takes account of information stability during writing3). Many TAMR media design parameters are related to each other in a complex manner. This new method can reveal the relationships between such parameters as anisotropy, Curie temperature, thickness and writing temperature. In our earlier work, we dealt with a hypothetical medium in which the magnetization, anisotropy, and Curie temperature are independent of each other. However, the magnetization and anisotropy are functions of the Curie temperature in actual materials.

We have also shown that the bit error rate (bER) after 10 years of archiving in granular media is a function of the grain number per bit $n$ and the standard deviation of the grain size $\sigma_{\mathrm{D}}$ as well as the thermal stability factor $\mathrm{TSF}_{10}{ }^{4}$.

In this study, we simulated our media design for 4 Tbpsi TAMR with this new method. We also considered improvement of the design method, the dependence of the magnetic properties on the Curie temperature, and bER dependence on $\mathrm{TSF}_{10}, n$, and $\sigma_{\mathrm{D}}$. As a result, we determined the anisotropy constant required for 4 Tbpsi TAMR.

\section{Heat Transfer Simulation}

To deduce the required anisotropy constant, we must first determine the thermal gradient of media at the time of writing. A heat transfer simulation was carried out using Poynting for Optics (Fujitsu Ltd.). Figure 1 is a schematic illustration of the structure of a medium that consists of four layers, that is, a recording layer $\mathrm{RL}(\mathrm{Fe}-\mathrm{Pt}$ base, $t \mathrm{~nm})$, interlayer $1 \mathrm{IL} 1(\mathrm{MgO}$ base, $5 \mathrm{~nm}$ ), interlayer $2 \mathrm{IL2}(\mathrm{Cr}$ base, $10 \mathrm{~nm}$ ), and a heat-sink layer HSL ( $\mathrm{Cu}$ base, $30 \mathrm{~nm})$. The $x, y$, and $z$ axes are the down-track, the cross-track, and the thickness directions, respectively. The writing temperature $T_{\mathrm{w}}$ is defined at the heat-spot edge and at the center of the RL layer in thickness direction, and the two positions of $T_{\mathrm{w}}$ in Fig. 1 are at a distance of $d_{\mathrm{w}}$ in the cross-track direction where $d_{\mathrm{w}}$ is defined as the heat-spot diameter. $T_{\max }$ is defined as the maximum surface temperature.

(a) Calculation conditions, (b) optical constants, and (c) thermal constants used in the simulation are summarized in Table 1 . Ambient temperature $T_{\mathrm{a}}$ is the maximum working temperature of the hard drive, and is assumed to be $330 \mathrm{~K}$.

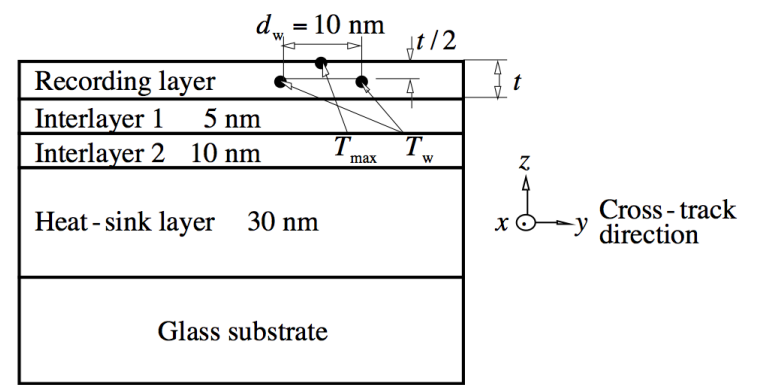

Fig. 1 Medium structure and definition of temperatures. 
A perfect conductor with a $12 \mathrm{~nm} \times 12 \mathrm{~nm}$ aperture is positioned $2 \mathrm{~nm}$ above the RL surface to generate a light spot with a diameter of several nanometers, and circularly polarized light at a wavelength of $780 \mathrm{~nm}$ is irradiated. Figure 2 shows the beam profile for the cross-track direction, and the light-spot diameter $d_{\mathrm{L}}$ (FWHM) is about $9.0 \mathrm{~nm}$. The profile for the down-track direction is almost the same as that in Fig. 2.

The calculated temperature profiles at the surface $z=0 \mathrm{~nm}$, and at 4 and $8 \mathrm{~nm}$ below the surface are shown in Fig. 3 for the cross-track direction. The two positions of $T_{\mathrm{w}}$ in Fig. 3 correspond with those in Fig. 1. A significant temperature gradient can be seen for the RL thickness direction. The temperature profile near $T_{\mathrm{w}}$ for the down-track direction is almost the same as that in Fig. 3.

Figure 4 (a) shows the calculated thermal gradients $\partial T / \partial x$ for the down-track direction and $\partial T / \partial y$ for the cross-track direction as a function of the $\mathrm{RL}$ thickness $t$, and $\partial T / \partial x \approx \partial T / \partial y$. The dependence of $T_{\max }$ on $t$ is shown in Fig. $4(\mathrm{~b})$.

Table 1 (a) Calculation conditions, (b) optical constants, and (c) thermal constants for heat transfer simulation.

(a)

\begin{tabular}{l|c}
\hline Wavelength $\lambda(\mathrm{nm})$ & 780 \\
\hline Light-spot diameter $d_{\mathrm{L}}(\mathrm{nm})$ & 9.0 \\
Heat - spot diameter $d_{\mathrm{w}}(\mathrm{nm})$ & 10 \\
Linear velocity $v(\mathrm{~m} / \mathrm{s})$ & 10 \\
Ambient temperature $T_{\mathrm{a}}(\mathrm{K})$ & 330 \\
\hline
\end{tabular}

(b)

\begin{tabular}{l|cc}
\hline & Refractive index & Extinction coefficient \\
\hline Recording layer & 3 & 4 \\
\hline Interlayer 1 & 1.73 & 0 \\
\hline Interlayer 2 & 4.11 & 4.35 \\
\hline Heat - sink layer & 0.242 & 4.85 \\
\hline Glass substrate & 1.5 & 0 \\
\hline
\end{tabular}

(c)

\begin{tabular}{|c|c|c|}
\hline & Specific heat & Thermal conductivity \\
\hline Recording layer & $\begin{array}{l}3 \mathrm{~J} /\left(\mathrm{cm}^{3} \mathrm{~K}\right) \\
200 \mathrm{~J} /(\mathrm{kgK})\end{array}$ & $1 \mathrm{~W} /(\mathrm{cmK})$ \\
\hline Interlayer 1 & $\begin{array}{l}2 \mathrm{~J} /\left(\mathrm{cm}^{3} \mathrm{~K}\right) \\
500 \mathrm{~J} /(\mathrm{kgK})\end{array}$ & $0.5 \mathrm{~W} /(\mathrm{cmK})$ \\
\hline Interlayer 2 & $\begin{array}{l}2.5 \mathrm{~J} /\left(\mathrm{cm}^{3} \mathrm{~K}\right) \\
360 \mathrm{~J} /(\mathrm{kgK})\end{array}$ & $1 \mathrm{~W} /(\mathrm{cmK})$ \\
\hline Heat - sink layer & $\begin{array}{l}4 \mathrm{~J} /\left(\mathrm{cm}^{3} \mathrm{~K}\right) \\
440 \mathrm{~J} /(\mathrm{kgK})\end{array}$ & $4 \mathrm{~W} /(\mathrm{cmK})$ \\
\hline Glass substrate & $\begin{array}{c}2 \mathrm{~J} /\left(\mathrm{cm}^{3} \mathrm{~K}\right) \\
1000 \mathrm{~J} /(\mathrm{kgK})\end{array}$ & $0.01 \mathrm{~W} /(\mathrm{cmK})$ \\
\hline
\end{tabular}

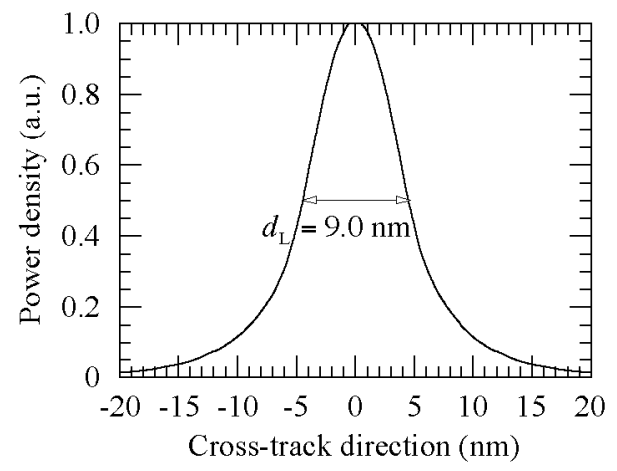

Fig. 2 Beam profile for the cross-track direction where the light-spot diameter $d_{\mathrm{L}}$ (FWHM) is about $9.0 \mathrm{~nm}$.

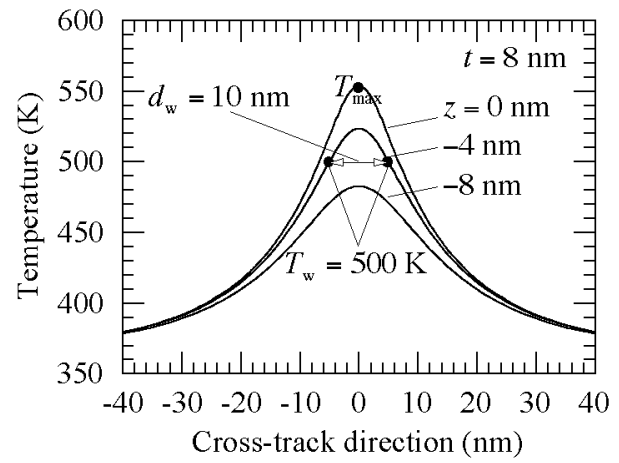

Fig. 3 Temperature profile for the cross-track direction where the heat-spot diameter $d_{\mathrm{w}}$ is $10 \mathrm{~nm}$ and writing temperature $T_{\mathrm{w}}$ is $500 \mathrm{~K}$.

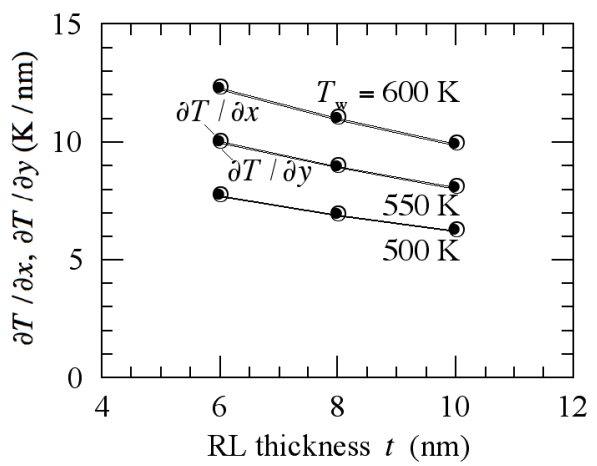

(a)

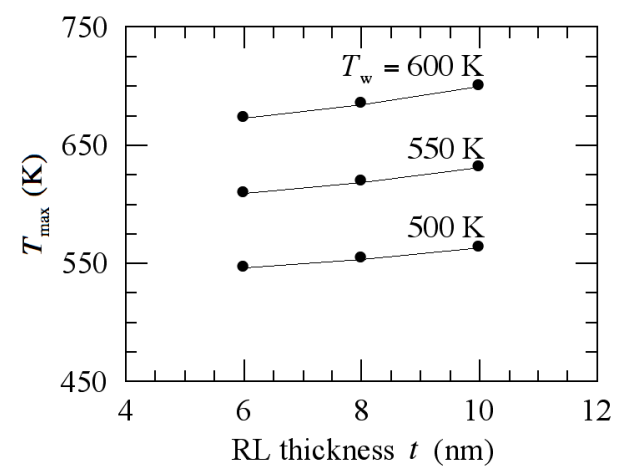

(b)

Fig. 4 (a) Thermal gradients $\partial T / \partial x$ for down-track direction and $\partial T / \partial y$ for cross-track direction, and (b) maximum surface temperature $T_{\max }$ as a function of RL thickness $t$. 


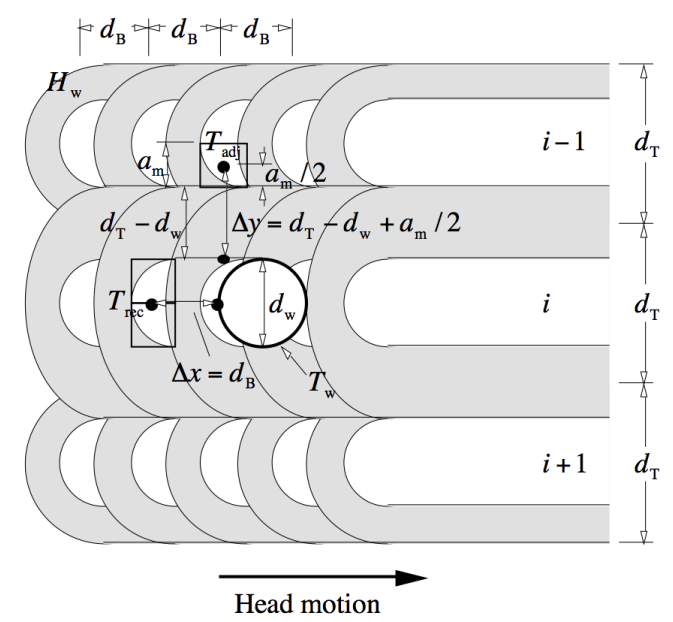

(a)

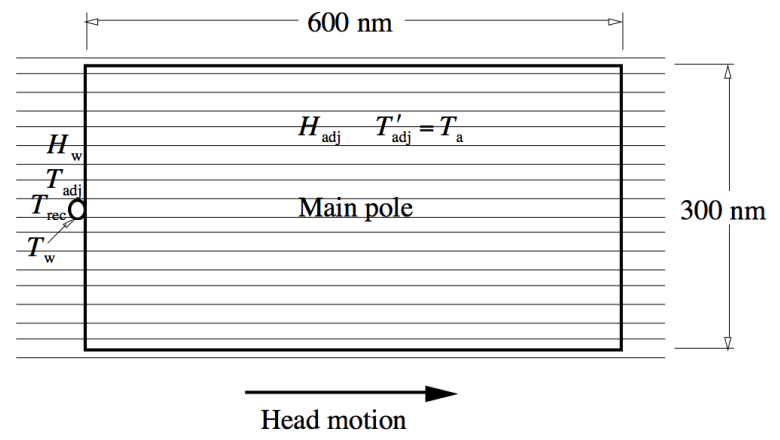

(b)

Fig. 5 Schematic illustrations of (a) writing position and (b) writing-head configuration.

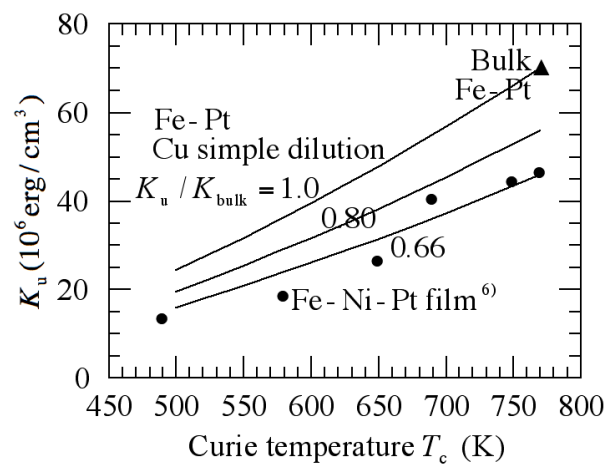

Fig. 6 Anisotropy constant $K_{\mathrm{u}}$ as a function of Curie temperature $T_{\mathrm{c}}$ for Fe-Pt systems.

As $t$ increases, the thermal gradient becomes smaller, and the maximum surface temperature becomes higher since the thick RL works adiabatically.

\section{Media Design Calculation Method}

Figure 5 (a) is a schematic illustration of the area near a writing position. The medium was assumed to be granular. A head field $H_{\mathrm{w}}$ is applied to a wide area including the writing position. The circle denoted $T_{\mathrm{w}}$ is an isothermal of $T_{\mathrm{w}}$ heated by the light, and $d_{\mathrm{w}}$ is the heat-spot diameter as shown in Fig. 1. The bit area $S=d_{\mathrm{B}} \times d_{\mathrm{T}}$ is fixed where $d_{\mathrm{B}}$ and $d_{\mathrm{T}}$ are the bit pitch and track pitch, respectively.

The white regions indicate upward or downward magnetization, and the gray regions indicate the magnetization transition. This figure shows the situation after many rewriting operations on the $i$ th track. The transition region spread to adjacent tracks as a result of the repetition of rewriting. Therefore, the stability of information on adjacent tracks during rewriting should be considered. The maximum temperature at which information can be held at an adjacent track is defined as $T_{\text {adj }}$. The distance $\Delta y$ from the isothermal $T_{\mathrm{w}}$ to the position of $T_{\text {adj }}$ was assumed to be $\Delta y=d_{\mathrm{T}}-d_{\mathrm{w}}+a_{\mathrm{m}} / 2$, where $a_{\mathrm{m}}$ is the mean grain size. Then $\left(T_{\mathrm{w}}-T_{\mathrm{adj}}\right) / \Delta y \equiv \Delta T / \Delta y$ is the minimum thermal gradient required by the medium for the cross-track direction, and it should be smaller than the thermal gradient $\partial T / \partial y$ calculated by the heat transfer simulation. $T_{\text {adj }}$ can be calculated from the thermal stability factor as mentioned in 4.4.

Of course, the minimum thermal gradient required by the medium for the down-track direction $\Delta T / \Delta x$ should also be considered. The maximum temperature that can hold the information recorded in 1 bit former during writing is defined as $T_{\mathrm{rec}}$. The distance $\Delta x$ from the isothermal $T_{\mathrm{w}}$ to the position of $T_{\text {rec }}$ was assumed to be the bit pitch $\Delta x=d_{\mathrm{B}}$. Then $\Delta T / \Delta x$ $=\left(T_{\mathrm{w}}-T_{\text {rec }}\right) / \Delta x$ should be smaller than the thermal gradient $\partial T / \partial x$ calculated by the heat transfer simulation. $T_{\text {rec }}$ can also be calculated from the thermal stability factor as mentioned in 4.3.

$d_{\mathrm{B}}, d_{\mathrm{T}}$, and the bit aspect ratio $d_{\mathrm{T}} / d_{\mathrm{B}}$ can be decided by $\Delta T / \Delta x=\Delta T / \Delta y$ since $\partial T / \partial x \approx \partial T / \partial y$ as shown in Fig. 4 (a). Since $T_{\text {rec }}$ and $T_{\text {adj }}$ are almost constant in spite of a few changes of $\Delta x$ as mentioned in 4.1 ,

$$
\frac{T_{\mathrm{w}}-T_{\text {rec }}}{T_{\mathrm{w}}-T_{\text {adj }}}=\frac{\Delta x}{\Delta y}=\frac{d_{\mathrm{B}}}{d_{\mathrm{T}}-d_{\mathrm{w}}+a_{\mathrm{m}} / 2} \equiv C
$$

is constant. Thus we can obtain $d_{\mathrm{B}}$ and $d_{\mathrm{T}}$ :

$$
d_{\mathrm{B}}=\frac{-C\left(d_{\mathrm{w}}-a_{\mathrm{m}} / 2\right)+\sqrt{C^{2}\left(d_{\mathrm{w}}-a_{\mathrm{m}} / 2\right)^{2}+4 C S}}{2}
$$

and

$$
d_{\mathrm{T}}=\frac{S}{d_{\mathrm{B}}} .
$$

The head field around the position at $T_{\mathrm{w}}, T_{\text {rec }}$ or $T_{\text {adj }}$ is $H_{\mathrm{w}}$, and $H_{\mathrm{w}}$ is determined from

$$
H_{\mathrm{w}}=16 \mathrm{kOe} \times \frac{M_{\mathrm{s}}(300 \mathrm{~K})}{1000 \mathrm{emu} / \mathrm{cm}^{3}}
$$

taking the stray field from the surrounding magnetization $M_{\mathrm{s}}(300 \mathrm{~K})$ into consideration.

The writing-head configuration is shown in Fig. 5 (b). It is also assumed that the main-pole size of the head is $600 \mathrm{~nm}$ (down-track direction) $\times 300 \mathrm{~nm}$ (cross-track direction). The writing position as shown in Fig. 5 (a) is located on the trailing side of the main pole. The maximum temperature that can hold the information under the head field during rewriting is defined as $T_{\text {adj }}^{\prime}$, and $T_{\text {adj }}^{\prime}=T_{\mathrm{a}}$. From $T_{\text {adj }}^{\prime}=T_{\mathrm{a}}$, the 
maximum head field $H_{\text {adj }}$ that can hold the information can be deduced from the thermal stability factor as mentioned in 4.5, and $H_{\text {adj }}$ should be higher than $H_{\mathrm{w}}$ due to the geometrical restriction of the head field.

The user areal density and the bit area $S$ were assumed to be 4 Tbpsi and $140 \mathrm{~nm}^{2}$, respectively. The simulation was carried out for grain numbers per bit $n$ of $4,5,6$, and 7 . The non-magnetic spacing $\Delta$ between grains is $1 \mathrm{~nm}$, and the mean grain size $a_{\mathrm{m}}$ can be calculated from $\sqrt{S / n}-\Delta$.

The temperature dependence of the magnetization $M_{\mathrm{s}}$ was determined using the mean field theory ${ }^{5}$, and that of the anisotropy constant $K_{\mathrm{u}}$ was assumed to be proportional to $M_{\mathrm{s}}{ }^{2}$ ). The $M_{\mathrm{s}}$ of $\mathrm{Fe}-\mathrm{Pt}$ with a Curie temperature $T_{\mathrm{c}}$ of $770 \mathrm{~K}$ was assumed to be 1000 $\mathrm{emu} / \mathrm{cm}^{3}$ at $300 \mathrm{~K}$. And the $M_{\mathrm{s}}(300 \mathrm{~K})$ of the medium is reduced with reducing $T_{\mathrm{c}}$.

Both $T_{\mathrm{c}}$ and $K_{\mathrm{u}}$ should be designated for TAMR media design. $K_{\mathrm{u}}\left(T_{\mathrm{c}}\right)$ at room temperature is a function of $T_{\mathrm{c}}$ as shown in Fig. 6. The closed circles in the figure are an example experimental result for Fe-Ni-Pt films ${ }^{6}$. $\quad K_{\mathrm{u}}\left(T_{\mathrm{c}}, T\right)$ is also a function of temperature $T$. It is intrinsic that $K_{\mathrm{u}}$ is reduced by reducing $T_{\mathrm{c}}$ since $K_{\mathrm{u}}\left(T_{\mathrm{c}}=R T, T=R T\right)=0 \quad(R T$ : room temperature). The $K_{\mathrm{u}}$ value of bulk Fe-Pt was assumed to be $70 \mathrm{Merg} / \mathrm{cm}^{3}$, and is shown as a closed triangle in the figure. The $K_{\mathrm{u}}$ value of $\mathrm{Fe}-\mathrm{Pt}$ film is generally smaller than that of bulk Fe-Pt. Therefore, we introduce a parameter $K_{\mathrm{u}} / K_{\text {bulk }}$ that shows the intrinsic ratio of film $K_{\mathrm{u}}$ to bulk $K_{\mathrm{u}}$. The solid line is a result calculated using the mean field theory for $\mathrm{Fe}-\mathrm{Pt}$ by $\mathrm{Cu}$ simple dilution. The calculation result reproduces the experimental result well for $K_{\mathrm{u}} / K_{\text {bulk }}=0.66$. If the $K_{\mathrm{u}} / K_{\text {bulk }}$ values for the media are identical, the difficulties involved in media preparation are the same, even if the $K_{\mathrm{u}}$ values are different since $K_{\mathrm{u}}$ can be changed only by changing the composition, e.g., the $\mathrm{Ni}$ composition in ( $\mathrm{Fe}-\mathrm{Ni})-\mathrm{Pt}$ or the $\mathrm{Cu}$ composition in ( $\mathrm{Fe}-\mathrm{Pt}$ )- $\mathrm{Cu}$. On the other hand, increasing $K_{\mathrm{u}} / K_{\text {bulk }}$ is challenging. Therefore, it is necessary to design a smaller $K_{\mathrm{u}} / K_{\text {bulk }}$ medium.

\section{Thermal Stability Factor}

The thermal stability factor $K_{\beta}$ is generally a function of temperature $T$ and magnetic field $H$, and is expressed as

$$
K_{\beta \pm}(T, H)=\frac{K_{\mathrm{u}}(T) V}{k T}\left(1 \pm \frac{H}{H_{\mathrm{c}}(T)}\right)^{2},
$$

where $V=a^{2} \times t$ ( $a$ : grain size) and $k$ are the grain volume and the Boltzmann constant, respectively, and the upper plus sign corresponds to a case where $H$ is parallel to $M_{\mathrm{s}}$ and the lower minus sign that where $H$ is antiparallel to $M_{\mathrm{s}}$. The coercivity $H_{\mathrm{c}}$ is assumed to be equal to the anisotropy field $H_{\mathrm{k}}=2 K_{\mathrm{u}} / M_{\mathrm{s}}$.

\subsection{Determination of composition}

We simulated the media design using the mean field theory for $\mathrm{Fe}-\mathrm{Pt}$ by $\mathrm{Cu}$ simple dilution rather than using that for $\mathrm{Fe}-\mathrm{Pt}$ by the $\mathrm{Ni}$ substitution of $\mathrm{Fe}$ because the calculation was simpler.

First, the $\mathrm{Cu}$ composition $x$ in $\left(\mathrm{Fe}_{0.5} \mathrm{Pt}_{0.5}\right)_{1{ }_{x}} \mathrm{Cu}_{x}$ should be determined. $x$ can be determined unambiguously from the writing temperature $T_{\mathrm{w}}$ under a certain $K_{\mathrm{u}} / K_{\text {bulk }}$.

Writing completion is defined as the state in which the direction of the recorded magnetization is stable for a certain time $\tau^{\prime}$ under head field $H_{\mathrm{w}}$ at temperature $T_{\mathrm{w}}$. For example, $\tau^{\prime}$ is $\Delta x / v=(7.5 \mathrm{~nm})$ $/(10 \mathrm{~m} / \mathrm{s})=0.75 \mathrm{~ns}$ (linear velocity $v=10 \mathrm{~m} / \mathrm{s})$. Since $H_{\mathrm{w}}$ is parallel to $M_{\mathrm{s}}$, the thermal stability factor $K_{\beta+}\left(T_{\mathrm{w}}, H_{\mathrm{w}}\right)$ is given by

$$
\begin{gathered}
\frac{\tau^{\prime}}{\tau}=\frac{0.75 \times 10^{-9}}{3.2 \times 10^{8}}=\frac{\exp \left(K_{\beta+}\left(T_{\mathrm{w}}, H_{\mathrm{w}}\right)\right)}{\exp \left(\mathrm{TSF}_{10}\right)}, \text { then } \\
K_{\beta+}\left(T_{\mathrm{w}}, H_{\mathrm{w}}\right)=\mathrm{TSF}_{10}-\ln \left(\frac{\tau}{\tau^{\prime}}\right)=\mathrm{TSF}_{10}-40.6,
\end{gathered}
$$

where $\mathrm{TSF}_{10}$ is the thermal stability factor calculated using many bits ${ }^{7}$, in which each bit has $n$ grains and each grain is of various sizes, under the condition of $10^{-3}$ bit error rate. $\mathrm{TSF}_{10}$ is calculated statistically using grain-error probability $P=1-\exp \left(-f_{0} \tau \exp \left(-\mathrm{TSF}_{10} \cdot\left(a / a_{\mathrm{m}}\right)^{2}\right)\right) \quad\left(f_{0}=10^{11} \mathrm{~s}^{-1}\right.$ : attempt frequency) for exactly $\tau=10$ years of archiving. $\mathrm{TSF}_{10}$ is a function of the grain number per bit $n$ and the standard deviation of the grain size $\sigma_{\mathrm{D}}$, and $\mathrm{TSF}_{10}$ has no relation with $K_{\mathrm{u}}$ since $\mathrm{TSF}_{10}$ is calculated statistically. $\mathrm{TSF}_{10}$ is for the grain of mean size $a=a_{\mathrm{m}}$. Then $K_{\beta+}\left(T_{\mathrm{w}}, H_{\mathrm{w}}\right)$ becomes

$$
\begin{aligned}
& K_{\beta+}\left(T_{\mathrm{w}}, H_{\mathrm{w}}\right)=\frac{K_{\mathrm{u}}\left(T_{\mathrm{w}}\right) V_{\mathrm{m}}}{k T_{\mathrm{w}}}\left(1+\frac{H_{\mathrm{w}}}{H_{\mathrm{c}}\left(T_{\mathrm{w}}\right)}\right)^{2}, \\
& =\mathrm{TSF}_{10}-40.6
\end{aligned}
$$

where $V_{\mathrm{m}}=a_{\mathrm{m}}{ }^{2} \times t$ is the mean grain volume. From Eq. (4), the $\mathrm{Cu}$ composition $x$ can be determined.

If $\Delta x=6.5 \mathrm{~nm}, K_{\beta+}\left(T_{\mathrm{w}}, H_{\mathrm{w}}\right)=\mathrm{TSF}_{10}-40.7$, and the difference of 0.1 in $K_{\beta+}\left(T_{\mathrm{w}}, H_{\mathrm{w}}\right)$ corresponds to a temperature difference of less than $1 \mathrm{~K}$. Therefore, $K_{\beta+}\left(T_{\mathrm{w}}, H_{\mathrm{w}}\right)=\mathrm{TSF}_{10}-40.6$ is applicable in this simulation in spite of a few changes of $\Delta x$.

\subsection{Condition (1)}

We estimated from the following four conditions whether or not the media have the potential for TAMR. That is, information stability (1) for 10 years of archiving, (2) on the trailing side located 1 bit from the writing position during writing, (3) in an adjacent track during rewriting, and (4) under the main pole during rewriting. The limiting factor under these conditions determines the minimum $K_{\mathrm{u}} / K_{\text {bulk }}$.

The first condition, namely the information stability for 10 years archiving, is expressed using $K_{\beta \pm}\left(T_{\mathrm{a}}, H=0\right) \equiv K_{\beta 0}\left(T_{\mathrm{a}}\right)$ as 


$$
K_{\beta 0}\left(T_{\mathrm{a}}\right)=\frac{K_{\mathrm{u}}\left(T_{\mathrm{a}}\right) V_{\mathrm{m}}}{k T_{\mathrm{a}}}>\mathrm{TSF}_{10}
$$

\subsection{Condition (2)}

The second condition, namely the information stability on the trailing side located 1 bit from the writing position during writing, determines the minimum thermal gradient $\Delta T / \Delta x$ required by the medium for the down-track direction.

The grains on the trailing side from the position of $T_{\mathrm{w}}$ are exposed to the head field $H_{\mathrm{w}}$ during the cooling process after the writing has taken place for a certain time $\tau^{\prime}$ at a certain temperature. Of course, the information recorded in the grains should be preserved. The maximum temperature that can hold the information is $T_{\text {rec }}$ as defined in 3. Since $H_{\mathrm{w}}$ may be antiparallel to $M_{\mathrm{s}}, T_{\text {rec }}$ can be calculated using the thermal stability factor $K_{\beta-}\left(T_{\text {rec }}, H_{\text {w }}\right)$ as

$$
\begin{aligned}
& K_{\beta-}\left(T_{\text {rec }}, H_{\mathrm{w}}\right)=K_{\beta 0}\left(T_{\text {rec }}\right)\left(1-\frac{H_{\mathrm{w}}}{H_{\mathrm{c}}\left(T_{\mathrm{rec}}\right)}\right)^{2} . \\
& =\mathrm{TSF}_{10}-40.6
\end{aligned}
$$

Then $\Delta T / \Delta x=\left(T_{\mathrm{w}}-T_{\text {rec }}\right) / \Delta x$ should be smaller than the thermal gradient $\partial T / \partial x$ calculated by the heat transfer simulation.

\subsection{Condition (3)}

The third condition, namely the information stability in an adjacent track during rewriting, determines the minimum thermal gradient $\Delta T / \Delta y$ required by the medium for the cross-track direction.

The maximum temperature that can hold the information in an adjacent track is $T_{\text {adj }}$ as defined in 3 . Assuming that the maximum number of rewriting operations is $10^{4}, T_{\text {adj }}$ can be calculated using the thermal stability factor $K_{\beta-}\left(T_{\text {adj }}, H_{\mathrm{w}}\right)$ as follows:

$$
\begin{aligned}
& \frac{\tau^{\prime} \times 10^{4}}{\tau}=\frac{\exp \left(K_{\beta-}\left(T_{\mathrm{adj}}, H_{\mathrm{w}}\right)\right)}{\exp \left(\mathrm{TSF}_{10}\right)} \text {, then } \\
& K_{\beta-}\left(T_{\mathrm{adj}}, H_{\mathrm{w}}\right)=K_{\beta 0}\left(T_{\mathrm{adj}}\right)\left(1-\frac{H_{\mathrm{w}}}{H_{\mathrm{c}}\left(T_{\mathrm{adj}}\right)}\right)^{2} . \\
& =\mathrm{TSF}_{10}-31.4
\end{aligned}
$$

Then $\Delta T / \Delta y=\left(T_{\mathrm{w}}-T_{\text {adj }}\right) / \Delta y$ should be smaller than the thermal gradient $\partial T / \partial y$ calculated by the heat transfer simulation.

\subsection{Condition (4)}

The fourth condition, namely the information stability under the main pole during rewriting, determines the maximum head field $H_{\text {adj }}$ that can hold the information.

The grains in the adjacent tracks are exposed to the head field at each time of rewriting, for a certain time $\tau^{\prime \prime}$ at temperature $T_{\text {adj }}^{\prime}=T_{\mathrm{a}}$. From the assumption, $\tau^{\prime \prime}$ is $(600 \mathrm{~nm}) /(10 \mathrm{~m} / \mathrm{s})=60 \mathrm{~ns}$. The head field is simultaneously applied to about other 15 tracks at each time of rewriting.

Since the head field may be antiparallel to $M_{\mathrm{s}}$, $H_{\text {adj }}$ can be calculated using the thermal stability factor $K_{\beta-}\left(T_{\text {adj }}^{\prime}=T_{\text {a }}, H_{\text {adj }}\right)$ as follows:

$$
\begin{aligned}
& \frac{\tau^{\prime \prime} \times 10^{4} \times 15}{\tau}=\frac{\exp \left(K_{\beta-}\left(T_{\mathrm{a}}, H_{\mathrm{adj}}\right)\right)}{\exp \left(\mathrm{TSF}_{10}\right)}, \\
& K_{\beta-}\left(T_{\mathrm{a}}, H_{\mathrm{adj}}\right)=K_{\beta 0}\left(T_{\mathrm{a}}\right)\left(1-\frac{H_{\mathrm{adj}}}{H_{\mathrm{c}}\left(T_{\mathrm{a}}\right)}\right)^{2}, \text { then } \\
& =\mathrm{TSF}_{10}-24.3 \\
& H_{\text {adj }}=H_{\mathrm{c}}\left(T_{\mathrm{a}}\right)\left(1-\sqrt{\frac{\mathrm{TSF}_{10}-24.3}{K_{\beta 0}\left(T_{\mathrm{a}}\right)}}\right)
\end{aligned}
$$

The maximum head field $H_{\text {adj }}$ should be higher than $H_{\mathrm{w}}$.

\section{Results}

\subsection{Dependence of grain number per bit}

Table 2 summarizes our calculation results. The calculation parameters are grain number per bit $n$, the standard deviation of the grain size $\sigma_{\mathrm{D}}$, the RL thickness $t$, and the writing temperature $T_{\mathrm{w}}$. Since the optimum $\mathrm{Cu}$ composition in $\mathrm{Fe}-\mathrm{Pt}-\mathrm{Cu}$ should be adjusted for each parameter change, the calculation results for the $T_{\mathrm{c}}, M_{\mathrm{s}}, K_{\mathrm{u}}, H_{\mathrm{c}}$, and $K_{\mathrm{u}} V_{\mathrm{m}} / k T$ values at $300 \mathrm{~K}$ are different. $\mathrm{TSF}_{10}$ is the thermal stability factor for 10 years of archiving calculated with $n$ and $\sigma_{\mathrm{D}}$. Table 2 also shows the optimum $d_{\mathrm{B}}, d_{\mathrm{T}}$, and $d_{\mathrm{T}} / d_{\mathrm{B}}$ values.

First, we considered the required $K_{\mathrm{u}} / K_{\text {bulk }}$ value for a case where the grain number per bit $n$ is changed with a fixed areal density. The conditions of (1) $K_{\mathrm{u}} V_{\mathrm{m}} / k T\left(T_{\mathrm{a}}\right)>\mathrm{TSF}_{10}$ and (4) $H_{\text {adj }}>H_{\mathrm{w}}$ have some margin, and the limiting factors are conditions (2) $\Delta T / \Delta x<\partial T / \partial x$ and (3) $\Delta T / \Delta y<\partial T / \partial y$ (shown as (2), (3) $\Delta T / \Delta x(y)$ in the table since $\partial T / \partial x=\partial T / \partial y)$. These limiting factors determine the minimum $K_{\mathrm{u}} / K_{\text {bulk }}$.

For example, $\mathrm{TSF}_{10}$ is 63 , and $K_{\mathrm{u}} V_{\mathrm{m}} / k T\left(T_{\mathrm{a}}\right)$ is 97 for $n=4$. If the medium has $K_{\mathrm{u}} V_{\mathrm{m}} / k T\left(T_{\mathrm{a}}\right)=63$, it can archive information for 10 years. However, a medium with $K_{\mathrm{u}} V_{\mathrm{m}} / k T\left(T_{\mathrm{a}}\right)=63$ is not suitable for TAMR, and $K_{\mathrm{u}} V_{\mathrm{m}} / k T\left(T_{\mathrm{a}}\right)=97$ is necessary. If the thermal gradient $\partial T / \partial x(y)$ can be increased, for example to $13.5 \mathrm{~K} / \mathrm{nm}$, a medium with $K_{\mathrm{u}} V_{\mathrm{m}} / k T\left(T_{\mathrm{a}}\right)=$ 64 becomes suitable for TAMR, and $K_{\mathrm{u}} / K_{\text {bulk }}$ can be reduced to 0.55 from 0.87 . As a result, the minimum $K_{\mathrm{u}} / K_{\text {bulk }}$ value strongly depends on the thermal gradient $\partial T / \partial x(y)$. If the thermal gradient exceeds $13.5 \mathrm{~K} / \mathrm{nm}$, the limiting factors will change from conditions (2) and (3) to condition (1) or (4).

As $n$ decreases, the required $\mathrm{TSF}_{10}$ becomes larger. However, both the grain size $a_{\mathrm{m}}$ and the volume become larger. As a result, the required $K_{\mathrm{u}}$, and therefore the required $K_{\mathrm{u}} / K_{\text {bulk }}$, become smaller by decreasing $n^{7)}$. The minimum $n$ value can be determined by the $\mathrm{SN}$ ratio of the reproduced signal, 
that is the complex matter of signal processing.

Table 2 Calculation results of TAMR media design for various grain numbers per bit $n$.

\begin{tabular}{l|cccc}
\hline$n$ (grain $/ \mathrm{bit})$ & 4 & 5 & 6 & 7 \\
$\sigma_{\mathrm{D}}(\%)$ & 10 & 10 & 10 & 10 \\
$t(\mathrm{~nm})$ & 8 & 8 & 8 & 8 \\
$T_{\mathrm{w}}(\mathrm{K})$ & 500 & 500 & 500 & 500 \\
\hline$a_{\mathrm{m}}(\mathrm{nm})$ & 4.92 & 4.29 & 3.83 & 3.47 \\
$T_{\mathrm{c}}(\mathrm{K})$ & 532 & 536 & 542 & 544 \\
$M_{\mathrm{s}}(300 \mathrm{~K})\left(\mathrm{emu} / \mathrm{cm}^{3}\right)$ & 655 & 662 & 672 & 676 \\
$K_{\mathrm{u}}(300 \mathrm{~K})\left(10^{6} \mathrm{erg}^{3} \mathrm{~cm}^{3}\right)$ & 25 & 28 & 31 & 33 \\
$H_{\mathrm{c}}(300 \mathrm{~K})=H_{\mathrm{k}}(300 \mathrm{~K})(\mathrm{kOe})$ & 77 & 84 & 93 & 98 \\
$K_{\mathrm{u}} V_{\mathrm{m}} / k T(300 \mathrm{~K})$ & 118 & 99 & 88 & 77 \\
\hline $\mathrm{TSF}$ & $63\left(n, \sigma_{\mathrm{D}}\right)$ & 63 & 59 & 57 \\
$(1) K_{\mathrm{u}} V_{\mathrm{m}} / k T\left(T_{\mathrm{a}}\right)>\mathrm{TSF}_{10}$ & 97 & 82 & 74 & 64 \\
\hline$\partial T / \partial x(y)(\mathrm{K} / \mathrm{nm})$ & 6.9 & 6.9 & 6.9 & 6.9 \\
$(2),(3) \Delta T / \Delta x(y)(\mathrm{K} / \mathrm{nm})$ & 6.9 & 6.9 & 6.9 & 6.9 \\
\multicolumn{1}{c}{$<\partial T / \partial x(y)$} & & & & \\
\hline$H_{\mathrm{w}}(\mathrm{kOe})$ & 10.5 & 10.6 & 10.8 & 10.8 \\
$(4) H_{\text {adj }}(\mathrm{kOe})>H_{\mathrm{w}}$ & 27.2 & 27.1 & 27.5 & 26.6 \\
\hline$K_{\mathrm{u}} / K_{\text {bulk }}$ & 0.87 & 0.94 & 1.02 & 1.07 \\
\hline$d_{\mathrm{B}}(\mathrm{nm})$ & 7.48 & 7.21 & 7.02 & 6.80 \\
$d_{\mathrm{T}}(\mathrm{nm})$ & 18.7 & 19.4 & 20.0 & 20.6 \\
$d_{\mathrm{T}} / d_{\mathrm{B}}$ & 2.50 & 2.69 & 2.84 & 3.03 \\
\hline
\end{tabular}

\subsection{Dependence of standard deviation of grain} size

The dependence of the required $K_{\mathrm{u}} / K_{\text {bulk }}$ value on the standard deviation of the grain size $\sigma_{\mathrm{D}}$ is shown in Table 3. As $\sigma_{\mathrm{D}}$ increases, the required $\mathrm{TSF}_{10}$ becomes larger. Therefore, the required $K_{\mathrm{u}} / K_{\text {bulk }}$ becomes larger by increasing $\sigma_{\mathrm{D}}$.

\subsection{Dependence of RL thickness}

Although the grain volume increases by increasing the RL thickness $t$, the required $K_{\mathrm{u}} / K_{\text {bulk }}$ values are almost the same as those shown in Table 4.

Figure 7 shows the dependence of $\Delta T / \Delta x(y)$ on $K_{\mathrm{u}} / K_{\text {bulk }}$ for each $t$. As $K_{\mathrm{u}} / K_{\text {bulk }}$ increases, $\Delta T / \Delta x(y)$ becomes smaller. The dotted lines in the figure show $\partial T / \partial x(y)$ for each $t$, and $\Delta T / \Delta x(y)$ should be smaller than $\partial T / \partial x(y)$. If $\partial T / \partial x(y)$ is constant in spite of the change of $t, K_{\mathrm{u}} / K_{\text {bulk }}$ can be decreased by increasing $t$. However, $\partial T / \partial x(y)$ is reduced by increasing $t$ due to the adiabatic effect of RL. Therefore, increasing the thickness has little merit as regards reducing $K_{\mathrm{u}} / K_{\text {bulk }}$.

\subsection{Dependence of writing temperature}

The dependence of the required $K_{\mathrm{u}} / K_{\text {bulk }}$ on the writing temperature $T_{\mathrm{w}}$ is shown in Table 5 .

Figure 8 shows the dependence of $\Delta T / \Delta x(y)$ on $K_{\mathrm{u}} / K_{\text {bulk }}$ for each $T_{\mathrm{w}}$. The dotted lines in the figure show $\partial T / \partial x(y)$ for each $T_{\mathrm{w}}$. As $T_{\mathrm{w}}$ increases, $\partial T / \partial x(y)$ becomes larger as shown in Fig. 4 (a). Therefore, increasing the writing temperature $T_{\mathrm{w}}$ is effective in reducing $K_{\mathrm{u}} / K_{\text {bulk }}$.
Table 3 Calculation results of TAMR media design for various standard deviations of grain size $\sigma_{\mathrm{D}}$.

\begin{tabular}{|c|c|c|c|c|}
\hline$n$ (grain / bit) & 4 & 4 & 4 & 4 \\
\hline$\sigma_{\mathrm{D}}(\%)$ & 5 & 10 & 15 & 20 \\
\hline$t(\mathrm{~nm})$ & 8 & 8 & 8 & 8 \\
\hline$T_{\mathrm{w}}(\mathrm{K})$ & 500 & 500 & 500 & 500 \\
\hline$a_{\mathrm{m}}(\mathrm{nm})$ & 4.92 & 4.92 & 4.92 & 4.92 \\
\hline$T_{\mathrm{c}}(\mathrm{K})$ & 517 & 532 & 548 & 571 \\
\hline$M_{\mathrm{s}}(300 \mathrm{~K})\left(\mathrm{emu} / \mathrm{cm}^{3}\right)$ & 632 & 655 & 682 & 718 \\
\hline$K_{\mathrm{u}}(300 \mathrm{~K})\left(10^{6} \mathrm{erg} / \mathrm{cm}^{3}\right)$ & 20 & 25 & 31 & 40 \\
\hline$H_{\mathrm{c}}(300 \mathrm{~K})=H_{\mathrm{k}}(300 \mathrm{~K})(\mathrm{kOe})$ & 63 & 77 & 92 & 112 \\
\hline$K_{\mathrm{u}} V_{\mathrm{m}} / k T(300 \mathrm{~K})$ & 93 & 118 & 146 & 188 \\
\hline $\mathrm{TSF}_{10}=f\left(n, \sigma_{\mathrm{D}}\right)$ & 54 & 63 & 75 & 94 \\
\hline (1) $K_{\mathrm{u}} V_{\mathrm{m}} / k T\left(T_{\mathrm{a}}\right)>\mathrm{TSF}_{10}$ & 76 & 97 & 122 & 159 \\
\hline$\partial T / \partial x(y)(\mathrm{K} / \mathrm{nm})$ & 6.9 & 6.9 & 6.9 & 6.9 \\
\hline $\begin{array}{c}\text { (2), (3) } \Delta T / \Delta x(y)(\mathrm{K} / \mathrm{nm}) \\
<\partial T / \partial x(y)\end{array}$ & 6.9 & 6.9 & 6.9 & 6.9 \\
\hline$H_{\mathrm{w}}(\mathrm{kOe})$ & 10.1 & 10.5 & 10.9 & 11.5 \\
\hline (4) $H_{\text {adj }}(\mathrm{kOe})>H_{\mathrm{w}}$ & 22.8 & 27.2 & 31.4 & 36.4 \\
\hline$K_{\mathrm{u}} / K_{\text {bulk }}$ & 0.74 & 0.87 & 1.00 & 1.15 \\
\hline$d_{\mathrm{B}}(\mathrm{nm})$ & 7.21 & 7.48 & 7.67 & 7.85 \\
\hline$d_{\mathrm{T}}(\mathrm{nm})$ & 19.4 & 18.7 & 18.2 & 17.8 \\
\hline$d_{\mathrm{T}} / d_{\mathrm{B}}$ & 2.69 & 2.50 & 2.38 & 2.27 \\
\hline
\end{tabular}

Table 4 Calculation results of TAMR media design for various RL thicknesses $t$.

\begin{tabular}{l|ccc}
\hline$n$ (grain $/ \mathrm{bit})$ & 4 & 4 & 4 \\
$\sigma_{\mathrm{D}}(\%)$ & 10 & 10 & 10 \\
$t(\mathrm{~nm})$ & 6 & 8 & 10 \\
$T_{\mathrm{w}}(\mathrm{K})$ & 500 & 500 & 500 \\
\hline$a_{\mathrm{m}}(\mathrm{nm})$ & 4.92 & 4.92 & 4.92 \\
$T_{\mathrm{c}}(\mathrm{K})$ & 544 & 532 & 524 \\
$M_{\mathrm{s}}(300 \mathrm{~K})\left(\mathrm{emu} / \mathrm{cm}^{3}\right)$ & 675 & 655 & 643 \\
$K_{\mathrm{u}}(300 \mathrm{~K})\left(10^{6} \mathrm{erg}^{3} \mathrm{~cm}^{3}\right)$ & 28 & 25 & 24 \\
$H_{\mathrm{c}}(300 \mathrm{~K})=H_{\mathrm{k}}(300 \mathrm{~K})(\mathrm{kOe})$ & 82 & 77 & 74 \\
$K_{\mathrm{u}} V_{\mathrm{m}} / k T(300 \mathrm{~K})$ & 97 & 118 & 139 \\
\hline $\mathrm{TSF} F_{10}=f\left(n, \sigma_{\mathrm{D}}\right)$ & 63 & 63 & 63 \\
$(1) K_{\mathrm{u}} V_{\mathrm{m}} / k T\left(T_{\mathrm{a}}\right)>\mathrm{TSF}_{10}$ & 81 & 97 & 115 \\
\hline$\partial T / \partial x(y)(\mathrm{K} / \mathrm{nm})$ & 7.7 & 6.9 & 6.2 \\
$(2),(3) \Delta T / \Delta x(y)(\mathrm{K} / \mathrm{nm})$ & 7.7 & 6.9 & 6.2 \\
\multicolumn{1}{c|}{$<\partial T / \partial x(y)$} & & & \\
\hline$H_{\mathrm{w}}(\mathrm{kOe})$ & 10.8 & 10.5 & 10.3 \\
$(4) H_{\text {adj }}(\mathrm{kOe})>H_{\mathrm{w}}$ & 24.5 & 27.2 & 29.8 \\
\hline$K_{\mathrm{u}} / K_{\text {bulk }}$ & 0.90 & 0.87 & 0.86 \\
\hline$d_{\mathrm{B}}(\mathrm{nm})$ & 7.39 & 7.48 & 7.55 \\
$d_{\mathrm{T}}(\mathrm{nm})$ & 18.9 & 18.7 & 18.6 \\
$d_{\mathrm{T}} / d_{\mathrm{B}}$ & 2.56 & 2.50 & 2.46 \\
\hline & & &
\end{tabular}

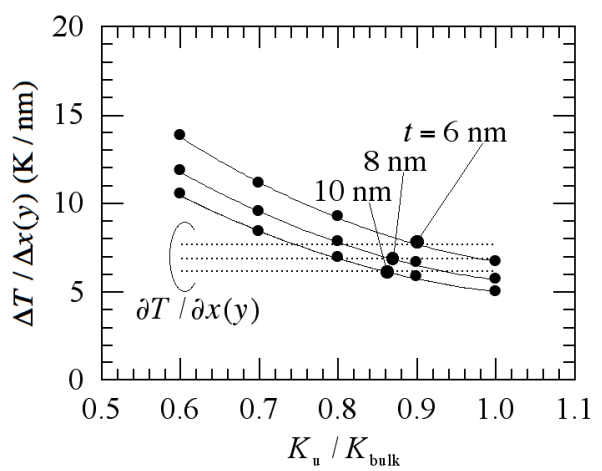

Fig. 7 Required thermal gradient $\Delta T / \Delta x(y)$ by medium as a function of intrinsic anisotropy constant $K_{\mathrm{u}} / K_{\text {bulk }}$. The calculation parameter is RL thickness $t$. 
Table 5 Calculation results of TAMR media design for various writing temperatures $T_{\mathrm{w}}$.

\begin{tabular}{l|ccc}
\hline$n$ (grain $/$ bit) & 4 & 4 & 4 \\
$\sigma_{\mathrm{D}}(\%)$ & 10 & 10 & 10 \\
$t(\mathrm{~nm})$ & 8 & 8 & 8 \\
$T_{\mathrm{w}}(\mathrm{K})$ & 500 & 550 & 600 \\
\hline$a_{\mathrm{m}}(\mathrm{nm})$ & 4.92 & 4.92 & 4.92 \\
$T_{\mathrm{c}}(\mathrm{K})$ & 532 & 583 & 634 \\
$M_{\mathrm{s}}(300 \mathrm{~K})\left(\mathrm{emu} / \mathrm{cm}^{3}\right)$ & 655 & 737 & 813 \\
$K_{\mathrm{u}}(300 \mathrm{~K})\left(10^{6} \mathrm{erg}^{3} / \mathrm{cm}^{3}\right)$ & 25 & 28 & 31 \\
$H_{\mathrm{c}}(300 \mathrm{~K})=H_{\mathrm{k}}(300 \mathrm{~K})(\mathrm{kOe})$ & 77 & 76 & 77 \\
$K_{\mathrm{u}} V_{\mathrm{m}} / k T(300 \mathrm{~K})$ & 118 & 131 & 146 \\
\hline $\mathrm{TSF} F_{10}=f\left(n, \sigma_{\mathrm{D}}\right)$ & 63 & 63 & 63 \\
$(1) K_{\mathrm{u}} V_{\mathrm{m}} / k T\left(T_{\mathrm{a}}\right)>\mathrm{TSF}$ & 97 & 112 & 126 \\
\hline$\partial T / \partial x(y)(\mathrm{K} / \mathrm{nm})$ & 6.9 & 9.0 & 11.0 \\
$(2),(3) \Delta T / \Delta x(y)(\mathrm{K} / \mathrm{nm})$ & 6.9 & 9.0 & 11.0 \\
\multicolumn{1}{c|}{$<\partial T / \partial x(y)(\mathrm{K} / \mathrm{nm})$} & & & \\
\hline$H_{\mathrm{w}}(\mathrm{kOe})$ & 10.5 & 11.8 & 13.0 \\
$(4) H_{\text {adj }}(\mathrm{kOe})>H_{\mathrm{w}}$ & 27.2 & 30.5 & 33.4 \\
\hline$K_{\mathrm{u}} / K_{\text {bulk }}$ & 0.87 & 0.76 & 0.69 \\
\hline$d_{\mathrm{B}}(\mathrm{nm})$ & 7.48 & 7.58 & 7.66 \\
$d_{\mathrm{T}}(\mathrm{nm})$ & 18.7 & 18.5 & 18.3 \\
$d_{\mathrm{T}} / d_{\mathrm{B}}$ & 2.50 & 2.43 & 2.39 \\
\hline & & & \\
\hline
\end{tabular}

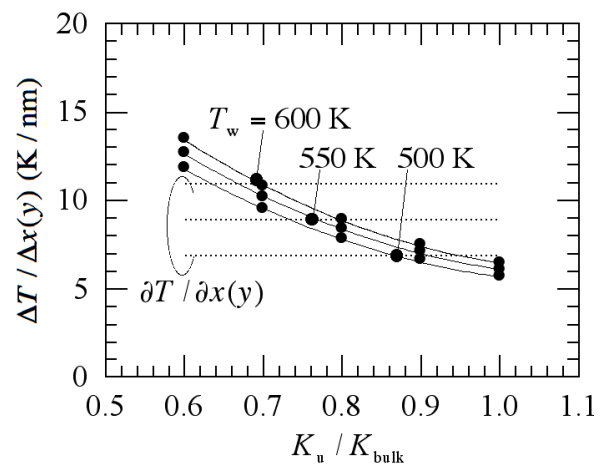

Fig. 8 Required thermal gradient $\Delta T / \Delta x(y)$ by medium as a function of intrinsic anisotropy constant $K_{\mathrm{u}} / K_{\text {bulk }}$. The calculation parameter is writing temperature $T_{\mathrm{w}}$.

\section{Conclusions}

The anisotropy constant $K_{\mathrm{u}}$ required for thermally assisted magnetic recording (TAMR) at 4 Tbpsi was evaluated by simulation. We introduced a parameter $K_{\mathrm{u}} / K_{\text {bulk }}$ that shows the intrinsic ratio of film $K_{\mathrm{u}}$ to bulk $K_{\mathrm{u}}$. It is necessary to design a smaller $K_{\mathrm{u}} / K_{\text {bulk }}$ medium.
The limiting factor whether or not the media have the potential for TAMR is the thermal gradient $\partial T / \partial x(y)$ calculated by a heat transfer simulation, and it determines the minimum $K_{\mathrm{u}} / K_{\text {bulk }}$.

(1) Dependence of grain number per bit $n$

As $n$ decreases, the thermal stability factor $\mathrm{TSF}_{10}$ calculated statistically becomes larger. However, the grain volume becomes larger. As a result, $K_{\mathrm{u}} / K_{\text {bulk }}$ becomes smaller by reducing $n$.

(2) Dependence of standard deviation of grain size $\sigma_{\mathrm{D}}$

As $\sigma_{\mathrm{D}}$ increases, $\mathrm{TSF}_{10}$ becomes larger. Therefore, $K_{\mathrm{u}} / K_{\text {bulk }}$ becomes larger with increasing $\sigma_{\mathrm{D}}$

(3) Dependence of recording layer (RL) thickness $t$

Although the grain volume increases with increasing $t, \partial T / \partial x(y)$ becomes smaller with increasing $t$ due to the adiabatic effect of RL. Therefore, the $K_{\mathrm{u}} / K_{\text {bulk }}$ values are almost the same.

(4) Dependence of writing temperature $T_{\mathrm{w}}$

As $T_{\mathrm{w}}$ increases, $\partial T / \partial x(y)$ becomes larger. Therefore, increasing $T_{\mathrm{w}}$ is effective in reducing $K_{\mathrm{u}} / K_{\text {bulk }}$.

Though optimum conditions can't be decided since there are many trade-off conditions, it can be point out that increasing $K_{\mathrm{u}} / K_{\text {bulk }}$ by an improvement of media preparation is necessary to realize 4 Tbpsi TAMR. An investigation of media structure with large $\partial T / \partial x(y)$ will also be effective to realize 4 Tbpsi TAMR.

Acknowledgements We acknowledge the support of the Storage Research Consortium (SRC), Japan.

\section{References}

1) S. H. Charap, P.-L. Lu, and Y. He: IEEE Trans. Magn., 33, 978 (1997).

2) T. Kobayashi, T. Kitayama, and Y. Fujiwara: J. Magn. Soc. Jpn., 35, 175 (2011).

3) A. Kikitsu, T. Kai, T. Nagase, and J. Akiyama: J. Appl. Phys., 97, 10P701 (2005).

4) T. Kobayashi, T. Kitayama, and Y. Fujiwara: J. Magn. Soc. Jpn., 36, 282 (2012).

5) M. Mansuripur, and M. F. Ruane: IEEE Trans. Magn., MAG-22, 33 (1986).

6) J. -U. Thiele, K. R. Coffey, M. F. Toney, J. A. Hedstrom, and A. J. Kellock: J. Appl. Phys., 91, 6595 (2002).

7) Y. Isowaki, T. Kobayashi, and Y. Fujiwara: J. Magn. Soc. Jpn., 38, 1 (2014).

Received Jun. 10, 2014; Accepted Nov. 14, 2014 